Mathématiques et sciences humaines
Mathematics and social sciences

183 | Automne 2008

Hommage à Georges-Th. Guilbaud

\title{
Un hommage (inédit) à G.-Th. Guilbaud par Pierre Gréco (1927-1988)
}

An unpublished homage to G.-Th. Guilbaud by Pierre Gréco (1927-1988)

\section{(2) OpenEdition}

Journals

Édition électronique

URL : http://journals.openedition.org/msh/10773

DOI : $10.4000 / \mathrm{msh} .10773$

ISSN : 1950-6821

Éditeur

Centre d'analyse et de mathématique sociales de l'EHESS

Édition imprimée

Date de publication : 14 décembre 2008

Pagination : 27-34

ISSN : 0987-6936

Référence électronique

"Un hommage (inédit) à G.-Th. Guilbaud par Pierre Gréco (1927-1988) », Mathématiques et sciences humaines [En ligne], 183 | Automne 2008, mis en ligne le 15 décembre 2008, consulté le 23 juillet 2020. URL : http://journals.openedition.org/msh/10773 ; DOI : https://doi.org/10.4000/msh.10773 


\author{
UN HOMMAGE (INÉDIT) À G.-Th. GUILBAUD \\ par Pierre GRÉCO (1927-1988)
}

Lors du colloque sur «L'à-peu-près » organisé à Urbino (14-16 juillet 1986) en l'honneur de G.-Th. Guilbaud, le psychologue Pierre Gréco, qui y participait à la fois comme ami et comme représentant de la présidence de l'EHESS, prononça, sans notes et fort brillamment selon son habitude, un discours qui impressionna fortement l'auditoire.

Après sa mort, on retrouva le manuscrit de l'essentiel - il manque au moins 3 pages - de ce discours.

Ce n'est qu'en 2004 que Monsieur Daniel Gilis - qui préparait son ouvrage Écrits en hommage à Pierre Gréco (Aléas (éd.), Lyon, mars 2008, 430 pages) - m'apprit cette trouvaille, et m'en communiqua une photocopie.

Je transmis aussitôt celle-ci à Guilbaud, qui en fut ému, et m'adressa quelques commentaires.

On trouvera ici la reproduction du texte manuscrit de Gréco (cf. p. 28-32), suivi de celle de l'essentiel des remarques, également manuscrites, de Guilbaud (cf. p. 33-34). 
(2)

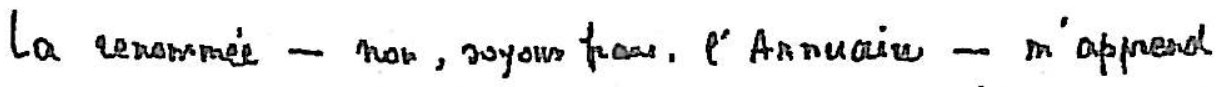

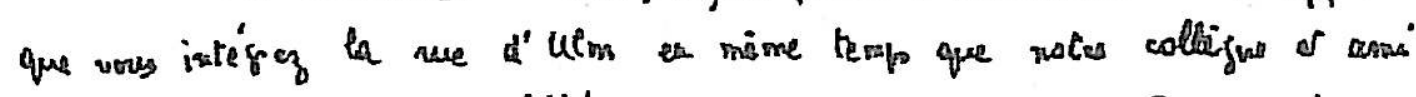

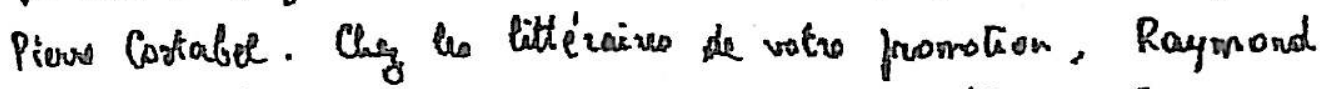

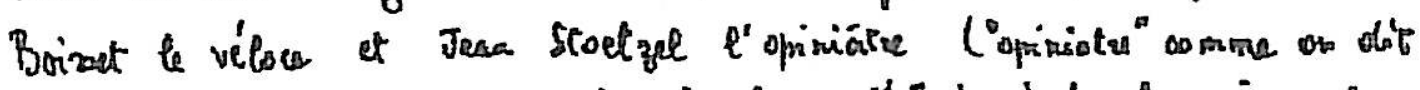

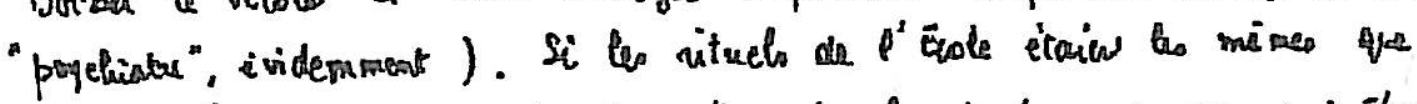

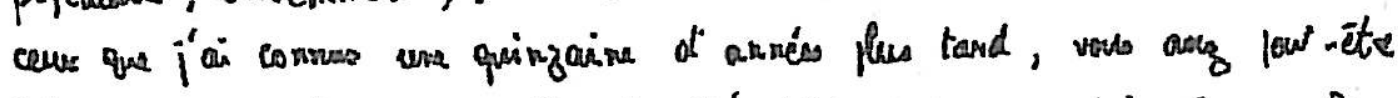

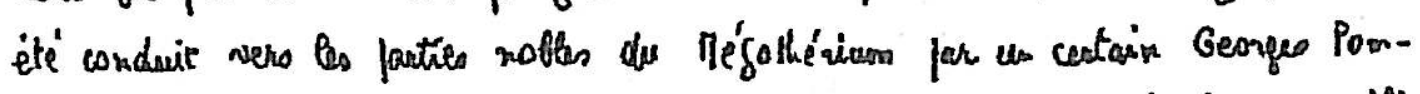



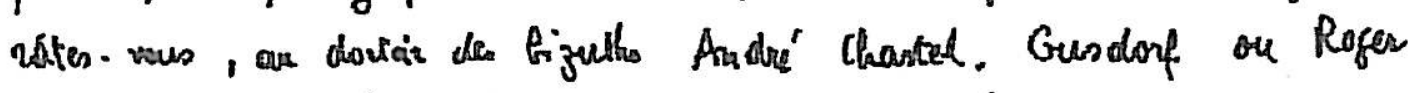

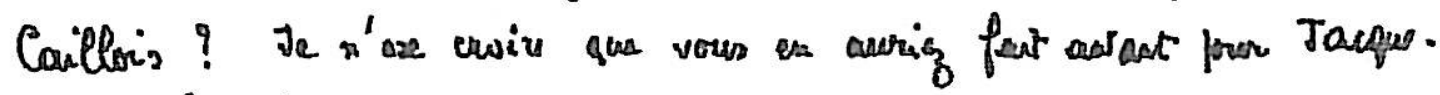
live of howitly 


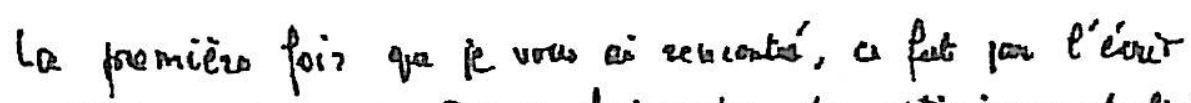

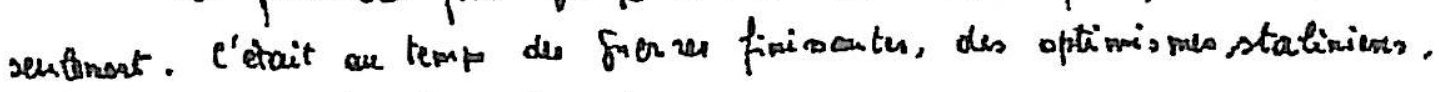
et des sounitures tereat us netrousés

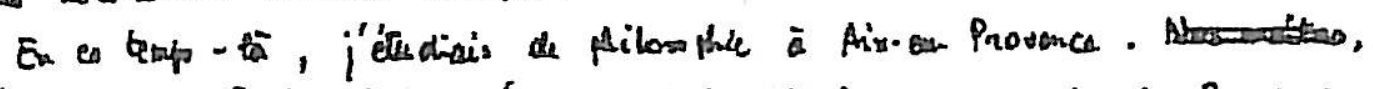

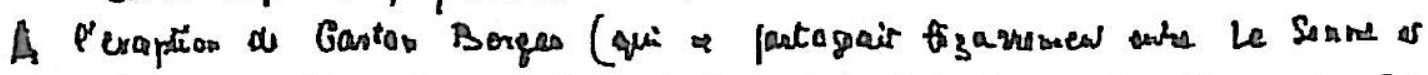

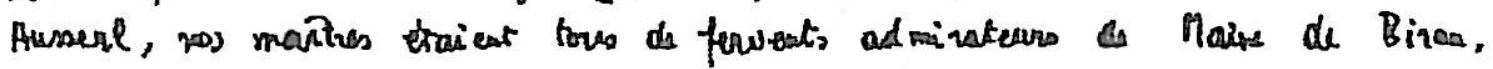



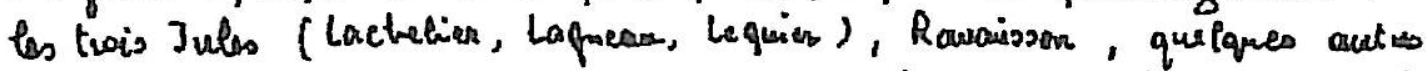

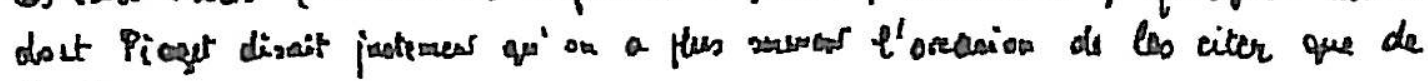
tis tive.

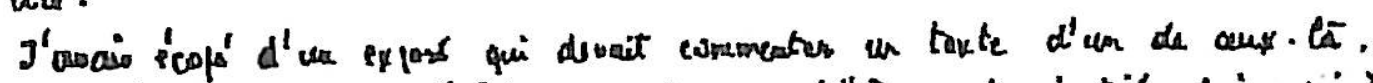

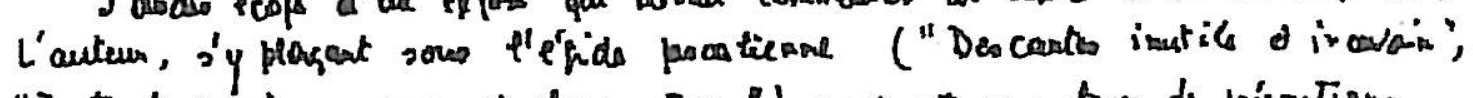

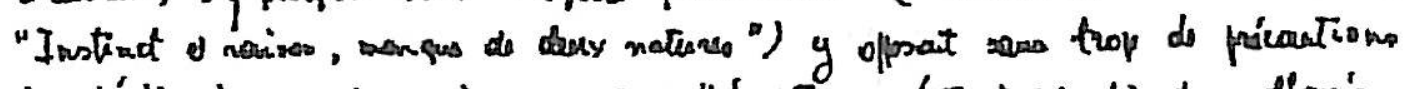

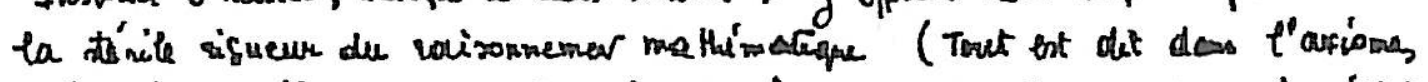

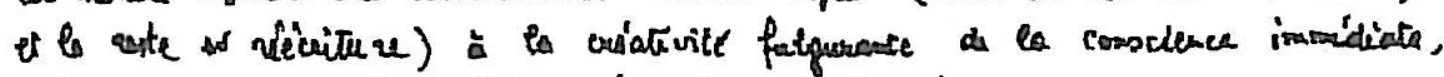

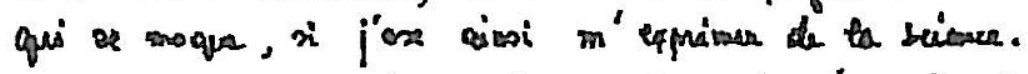

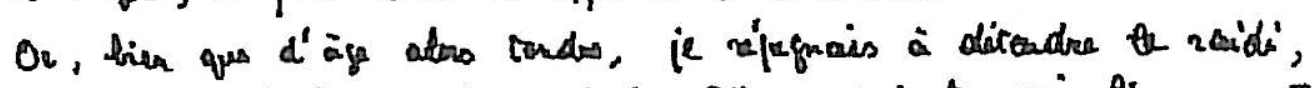

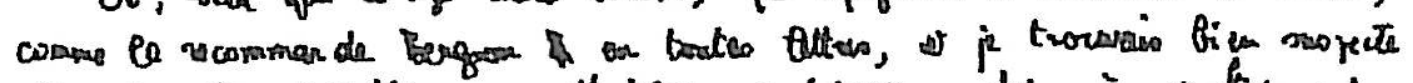

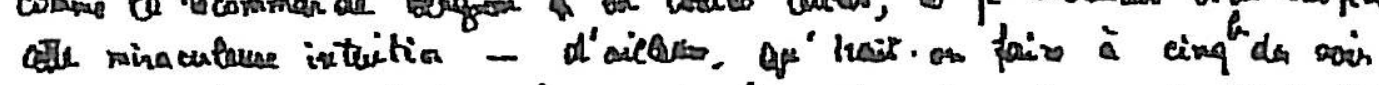
au coetar d'ua arere? La fréstere de lamadryodos s'y parainair proted.

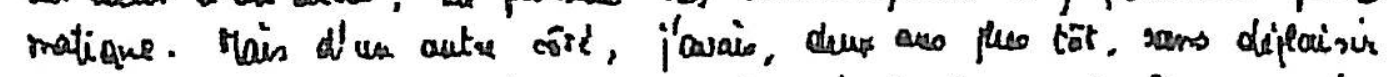



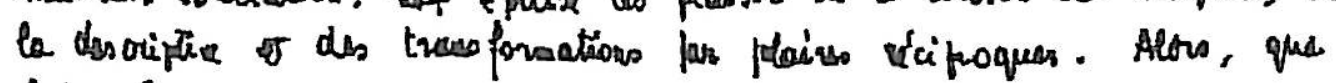
fain?

Ex Khägre à Tarocitle venair de débarepen un nouveau pobereur.

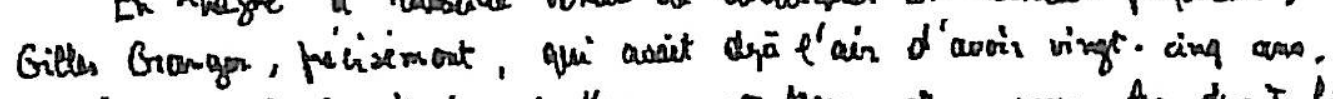

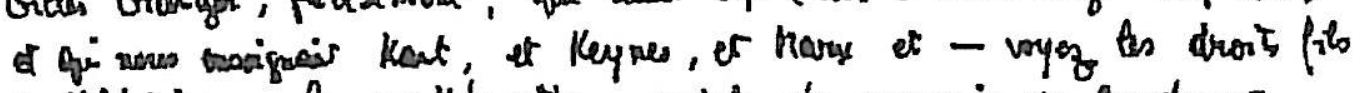
de l'hirtriu - Pa mathimatique rociole de manquis de Condoncer.

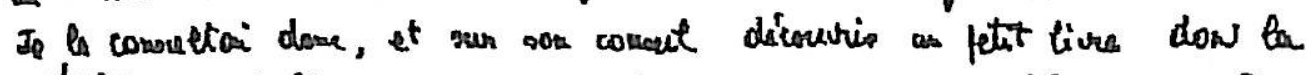

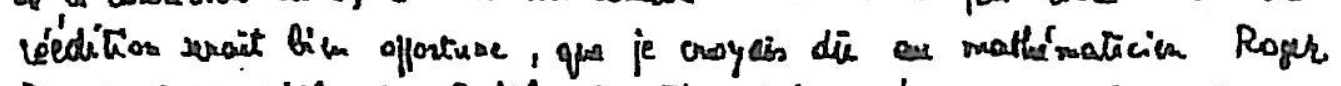

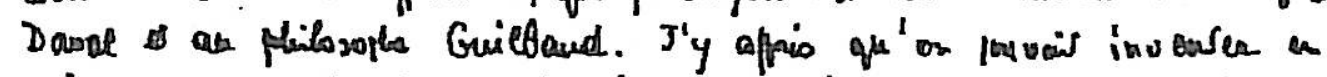

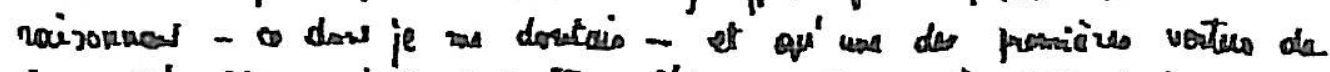

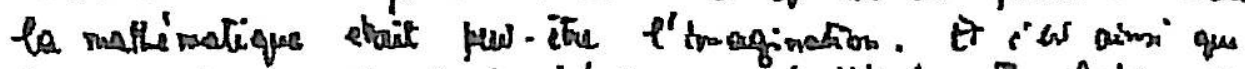

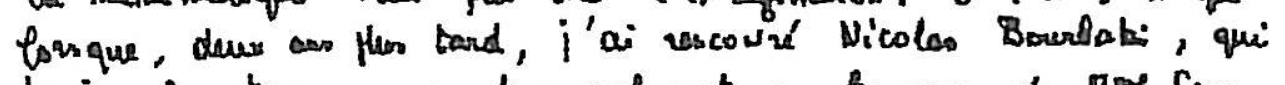

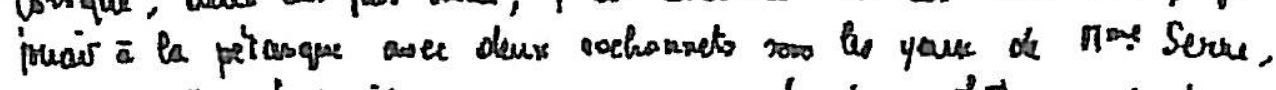

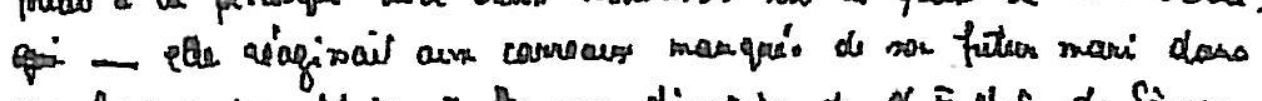

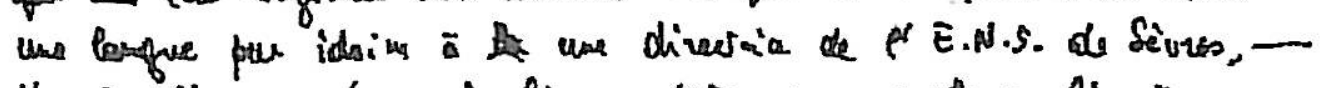

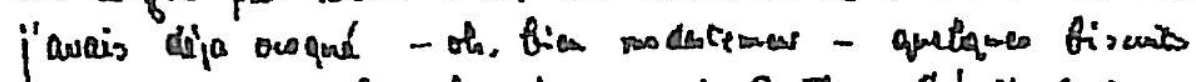

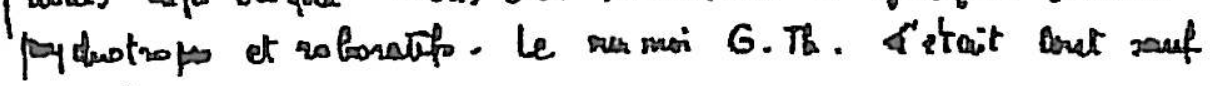
represit. 


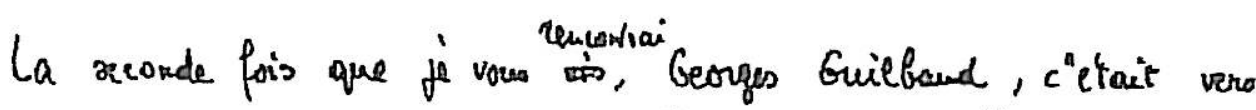
1955. Avec Piaget - nos, dizous: pour Piayes - je me livais à la räche

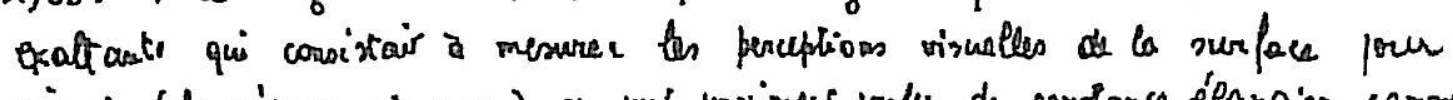

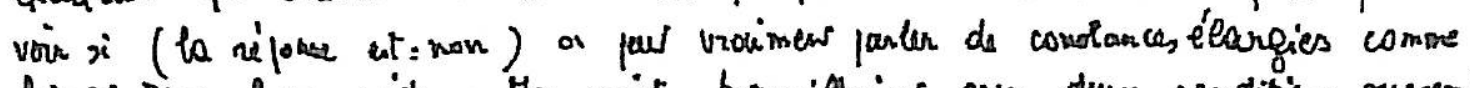

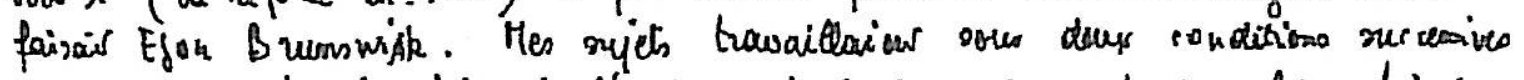

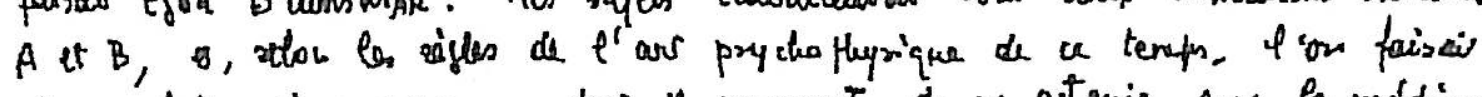
charque fors cing mesures, dow il converest de re retemir que la médiane.

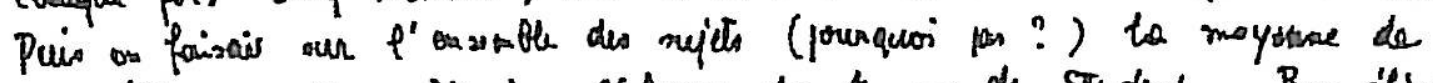

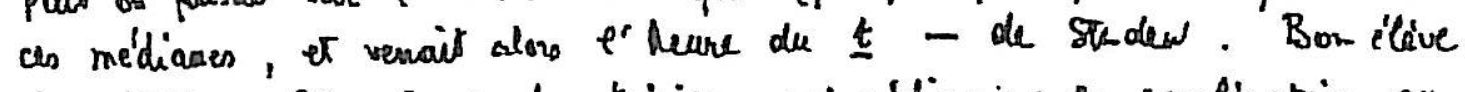

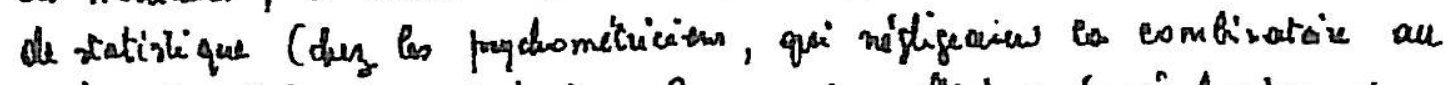
profit de l'équation de laplace. Gaves, des cofficiens éméáchariquen de

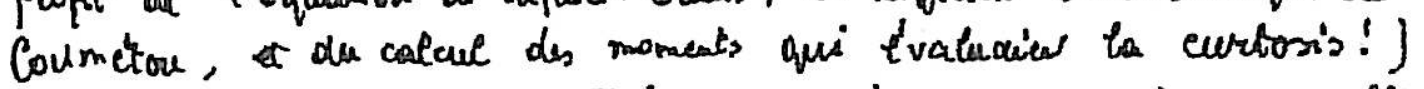
je entais ic fris de court. Fatloi vores roir, exposai mar probline,

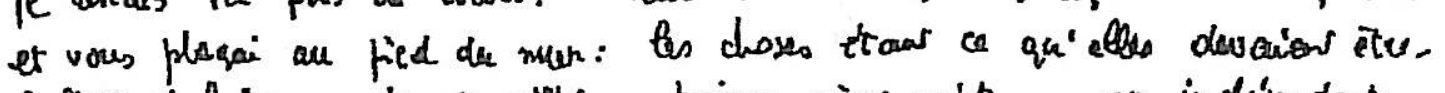

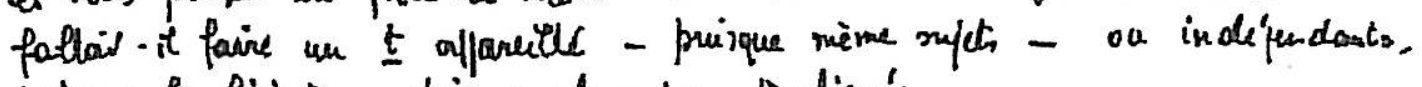
puirque to fijection stais quelque pee trafiquéc.

Vous m'avey écocté latiemaness, en sourriar, et, fiom sies vores

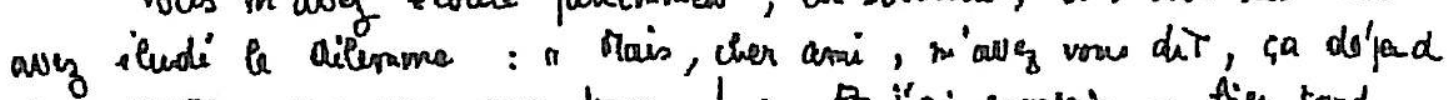

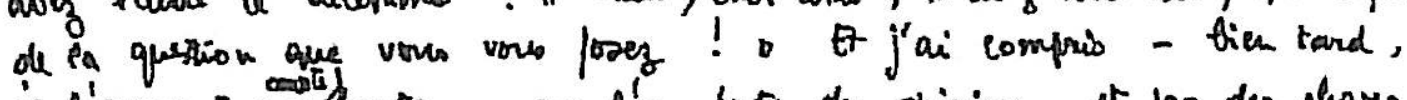

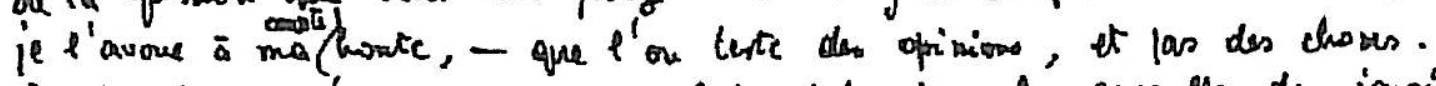

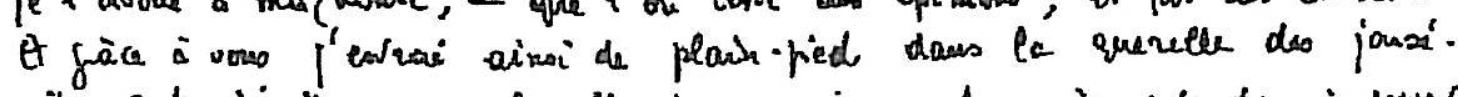

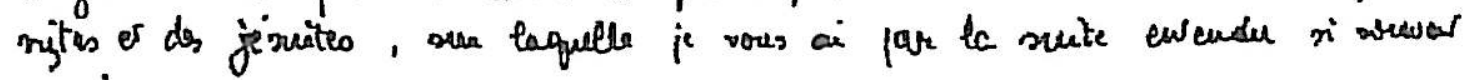
neweria.

Je me rousicas ausoi, an rempo to on la recherehe voulus se faine

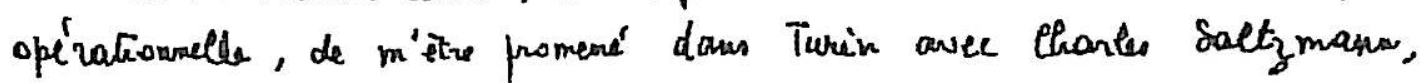

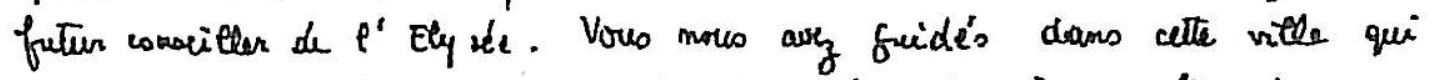

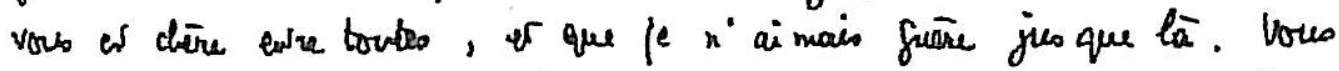

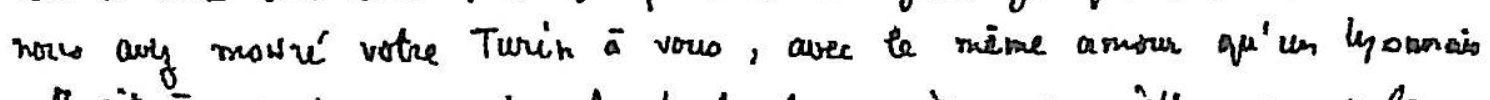

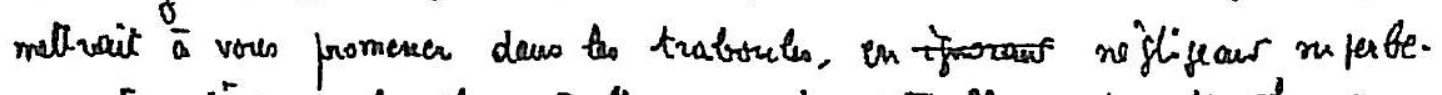

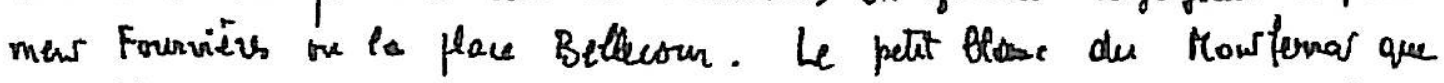

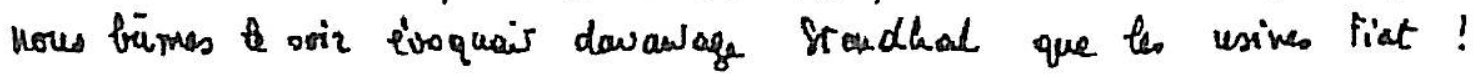


Vous owez ité thonucur de notra insticution. Oh certes, vous

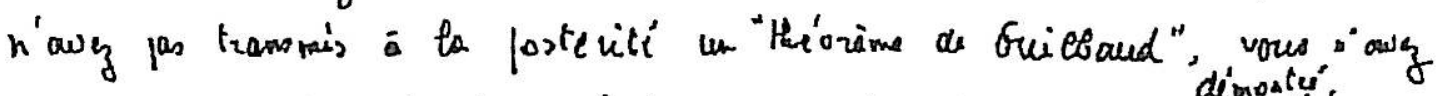

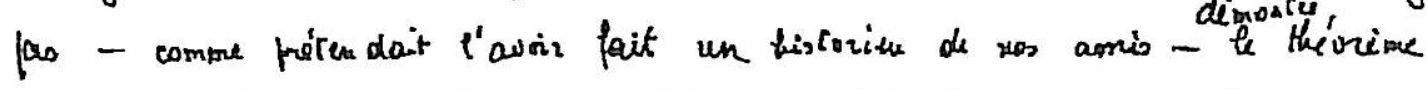
de Fermat (vérification faite, it o'étair vantí), fait pofresser la conjecture

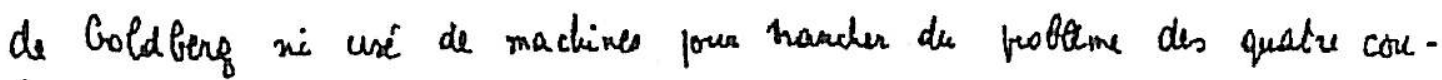
leurs, tonjours consroverse' que je sache.

Mais vous avey fait en sorte qu'aucus de vos acdeteurs n'a

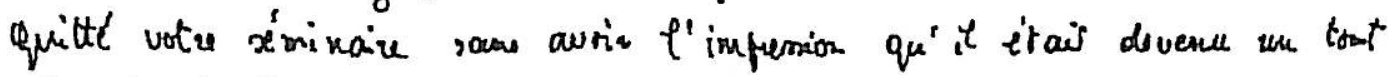
peter ples inteligest

I faut $l^{\prime}$ avoreer, vous asly été méconnus. Combier d'entre now, es hor de moirdres - Fernand, Flansais, Emmanuel et quelques autus... -

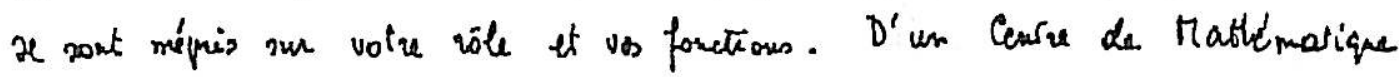



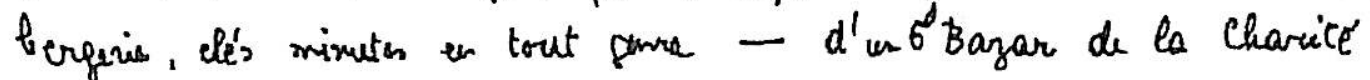
prope à résoudre nos émigmes, aloss qua vores, pampier pyromare. bous tentiy d'eteindere quolques incesolies dow cartains - et c'er heureuxavaiens étét otlumis volu main. Ils voulaier Benzécri, rores

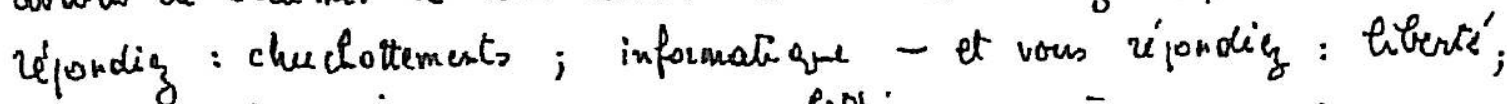

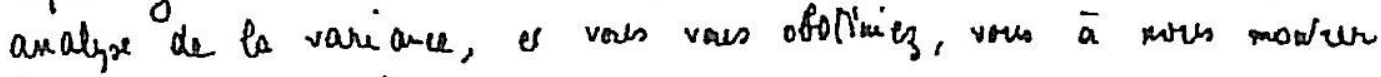
les variances de l'analyse.

Dass be didale de as rciences qui se disens sociales, et qui toriours

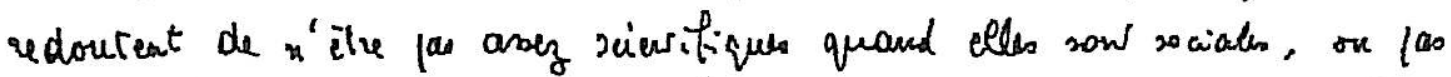

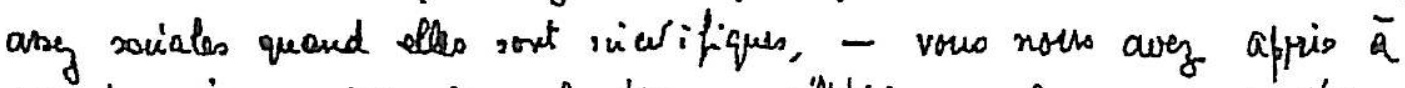

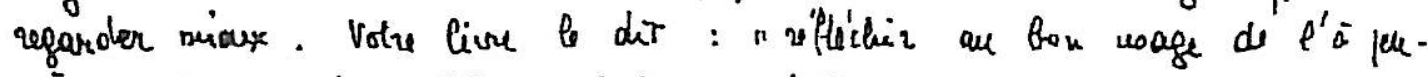
pés." Vous n'asez ste ni Aniane la folle, qui dir d'atler de

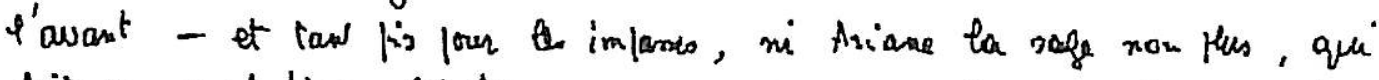
fait vite umbabixer crainte de 's égarer. Te vous imagive

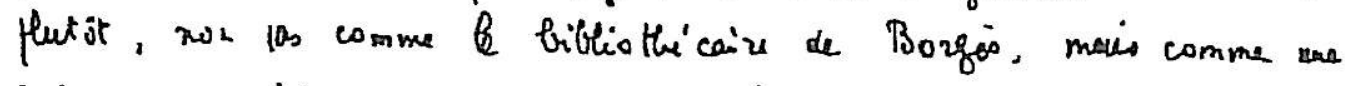
Ariane sams fit - meine rouge, un fit, sa fair des noesude parfors qu'ou ne rait defaire ove sus le papier - une Ariane qui feignait, hypoonite

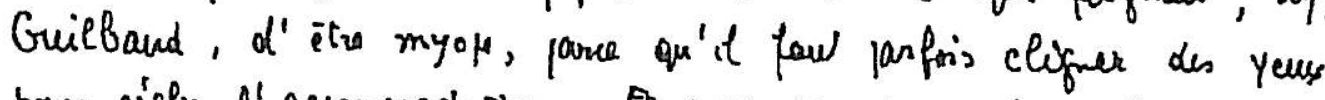
pour rifler l'accosomodacion. At nous sommes numbrcur à vous an

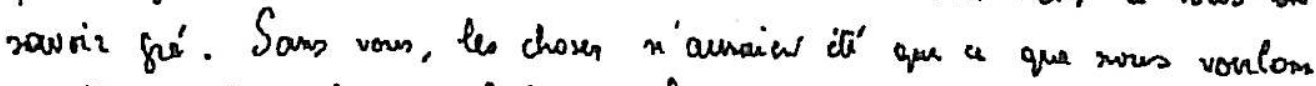
qu'elles roient : hatitus habituar ficor. 
Oui, l'lossneur de l'Ecoles. Y a-t-il aes monde une inctiturion

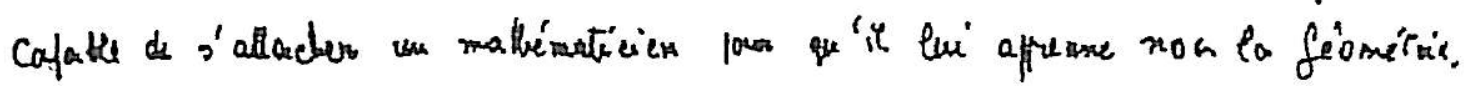
mais forem?

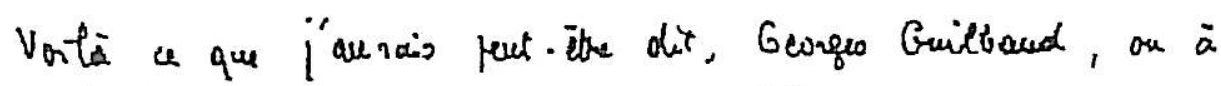

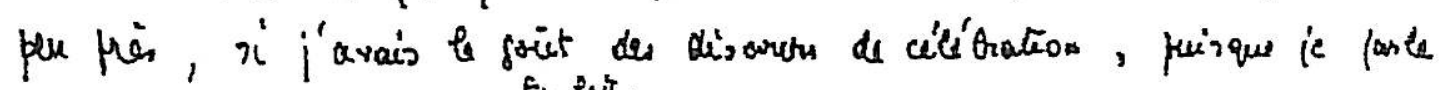

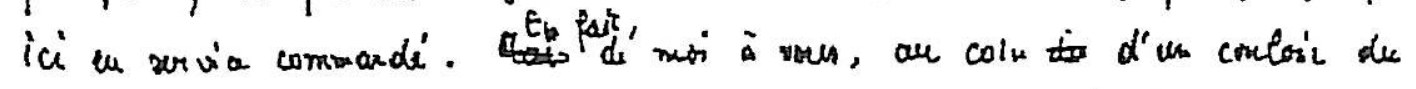

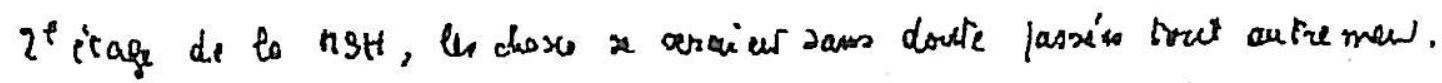
Mais quor, dwow une ouss docte assemblée, quand on a des moeuto à peu pis normale, ert.ce qu'on feut vous dive : "Je vals

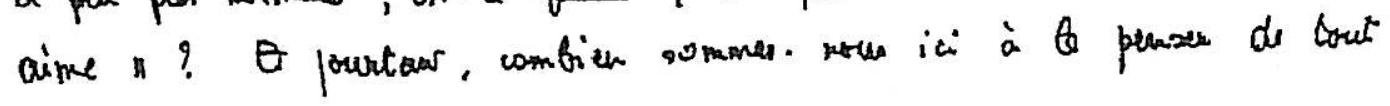
couer.

Je terminerai - be bous arteurs le recommonders - jar un

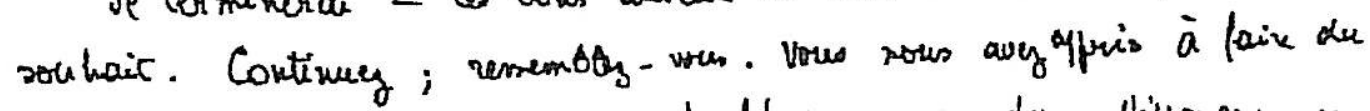

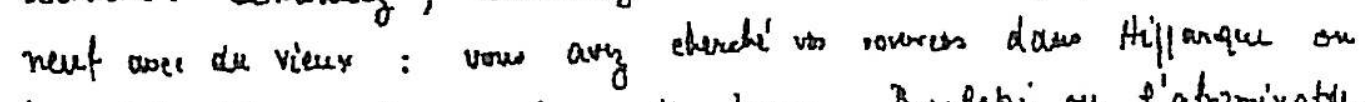
dam Gringalet, plutor que daess He lane, Bourbaki ou f'abomixable

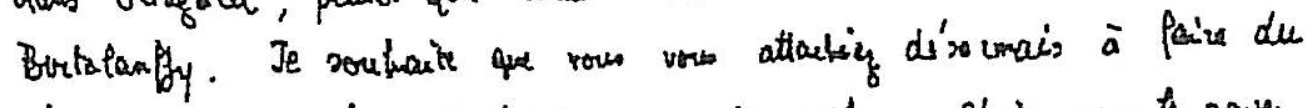
vieus avec de reeft. suremess, uns défirition crucivertale de Robor Sciprion gre Perec

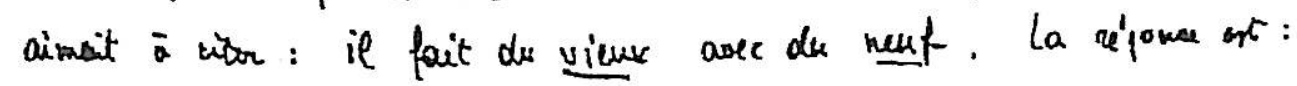
nonaginaire. 
3/ Huit fevilles a Lotot, numèrolés le $n \circ 8$ es bien le find : on $g$ lit to conclusion, destingie a attinver alte impression dorasis an funèbre (as nutice nècrolojique) qu'avait po donner le texte - conclusian qui me toudle, puisque, comme elle souhaite, f'ai c'tte int er dépassé les nonante annobs de vie.

Manqueir n'1, 5 et6. Porrquai?

qui a retrouva us papien, eroi'? Des psycholy gras me dites-vovi_ ce qui est un per rague.

quiz ècrir ce titre "Airesse a Gr.Th. G4 que je ne lis pol en manuscrit.

qu'g avaitil dans 1 fevilles manquants?

je devrais pouvair en dire quelquecliose epuisque j'di entend, la bi 2u discoun, lequl m'arait singulièrement freppoi.

Mas ma mèmoire est fort vièe!

Grece dair sans doute porlè de Condorcer; al te dit au dehot dè sa première rencontre drec Granger. Eh, je crais bien en ètre sür, ù èroqua't avsti le Centee Condercet: comme row k savea, il avair bien connu la Rve Richer, dont il appreaciat b mèmoire faustrollienne (il we le disain)

Ce texte, tel quil est ¿̀ présent, pourrat il interesserquelques lecterrs? J'hesite do repondre.

outre l'ironie subrile, difficile à saitir par endroits, à ya trip d'allusions qu'it conviendraird'édairer, si on ostait.

quelquen ex emple de ces bizarreries:

1) la rencontra de Turin (en jèumbre 1956)

d'avove qe, triente ans apres, g'evels oublie jusqu' a ce momeat oi il me reppelait que c'etait whe premieice rencoutre. Toul-est dir, mals do fagon elliptigua. Et qui pourtait siuteresuer ¿ de tels de'tills?

i) 4 dak Ariane: $k$ fil rouge qu-foir des noevds

Lil a voulu ne pas nommer alvi d̀qui nous peusions tous deer) 
4/301 la consultation (chej Piaget) ₹ propos du Es de Student. "en ne teste que des opinions". qui comprendra quil s'ajir do lenjage des Probabilitè (que d'eveuns d'obitineut s' dire subjectives) et pourquà l'pithe'h "bayesien" nest per ènoniè?

4) "chuchatementy" ditil, coutre Benzecri.

Incomprehentible pour la plupart de lectevrs.

(qvi peusere a Bergmon?) et particulieremont subtil.

s) qui entendrait l'hamadryale à cing hevres de sair? ef ity eu dureit d'atre encore:

6) qui oserait designer (en le nommonbs ce cox qui sout accosis de n'avir rian caupris à la Mathemachigre Locidele! Fernand, Frangcis, Emmanuel.

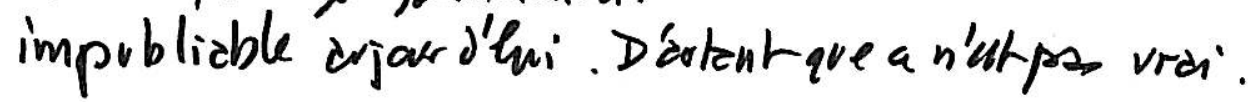

Publier vntel texte (incomplay oan notre Revive, proporet vous. Je n'ai pa Sevis à donner. Je reppelletai seulemeat axp responsably dela $R_{e} d_{a c t i o n} q u e$ nos lecterrs attendeut bes texter scientifigres et non 10 teutimast. Sile $y$ tieaneat quoul mems, quab att ndeult quage sois morr (cad ne tarderd pa Mop), lo ai je serai, je n'durai plu f'occasionde m'irriter.

Et pour vos psychologess (quivous out doune' le texte) its peoveat faire ce quils veuleut en décorper des morcosu de a texte incoupplet par agremeuter un portroit un portrdit de Pierre Grees (mals qu'in sonuout un fee simile de l'éaiturs!)

Pardonney tource bavardoge. Es cooyet moi votru $A_{m i}$. Merciencoure. g. the. 